\title{
Evaluation of mitochondrial function and metabolic reprogramming during tumor progression in a cell model of skin carcinogenesis
}

\author{
Carmen G. Vallejo ${ }^{a, b, c}$, Alberto Cruz-Bermúdez ${ }^{a, b, c}$, Paula Clemente ${ }^{a, b, c}$, Rosana \\ Hernández-Sierra ${ }^{a, b, c}$, Rafael Garesse ${ }^{a, b, c, *}$ and Miguel Quintanillaa,d,* \\ a Instituto de Investigaciones Biomédicas "Alberto Sols" Consejo Superior de \\ Investigaciones Científicas (CSIC)-Universidad Autónoma de Madrid (UAM), \\ Madrid, Spain \\ b Centro de Investigación Biomédica en Red (CIBERER), Facultad de Medicina, \\ UAM, Madrid, Spain \\ c Instituto de Investigación Sanitaria Hospital 12 de Octubre (i+12), Madrid, \\ Spain \\ d Instituto de Investigación Hospital Universitario La Paz (idipaz), Madrid, \\ Spain
}

Corresponding author to handle the manuscript: Carmen G. Vallejo

Address: Instituto de Investigaciones Biomédicas Alberto Sols, CSIC-UAM, Arturo

Duperier 4, 28029-Madrid, Spain.

e-mail : cvallejo@iib.uam.es

Phone: 34-915854425

Fax : $\quad 34-915854401$

Corresponding authors at: Instituto de Investigaciones Biomédicas Alberto Sols,

CSIC-UAM, Arturo Duperier 4, 28029-Madrid, Spain.

E-mail addresses:

cvallejo@iib.uam.es; rafael.garesse@iib.uam.es; mquintanilla@iib.uam.es

\section{Keywords}

Mitochondria, glycolysis, metabolic reprogramming, ROS, carcinogenesis 


\section{Abstract}

Metabolic reprogramming from mitochondrial aerobic respiration to aerobic glycolysis is a hallmark of cancer. However, whether it is caused by a dysfunction in the oxidative phosphorylation pathway is still under debate. In this work, we have analyzed the bioenergetic cellular (BEC) index and the relative cell ability to grow in the presence of either galactose or glucose as sources of sugar (Gal/Glu index) of a system formed by four epidermal cell lines with increasing tumorigenic potentials, ranging from nontumorigenic to highly malignant. We find that the BEC index gradually decreases whereas the Gal/Glu index increases with tumorigenicity, indicating that a progressive metabolic adaptation to aerobic glycolysis occurs in tumor cells associated with malignancy. Interestingly, this metabolic adaptation does not appear to be caused by damaged respiration, since the expression and activity of components of the respiratory chain complexes were unchanged in the cell lines. Moreover, the corresponding mitochondrial ATP synthetic abilities of the cell lines were found similar. The production of reactive oxygen species was also measured. A shift in ROS generation was found when compared nontumorigenic with tumorigenic cell lines, the latter exhibiting about threefold higher ROS levels than nontumorigenic cells. This result indicates that oxidative stress is an early event during tumor progression. 


\section{Introduction}

In 1927, Otto Warburg put forward the hypothesis that cancer cells undergo a shift in their metabolism from mitochondrial aerobic respiration to glycolysis followed by lactic acid fermentation that would occur even under normoxic conditions. Warburg also proposed that this altered metabolism is caused by defects in the oxidative phosphorylation (OXPHOS) pathway [1]. A bioenergetic signature of cancer cells was proposed by Cuezva and collaborators who showed that the expression of mitochondrial and glycolytic enzymatic markers varies significantly when comparing human carcinomas with paired normal tissues [2]. These authors analyzed a wide variety of tumors, including liver, kidney, colon, breast, lung, gastric and esophageal carcinomas, and concluded that OXPHOS function is reduced during neoplastic transformation. They defined a bioenergetic cellular index, the BEC index, which could be used to estimate the mitochondrial status in human tumors. However, the concept that the respiration of cancer cells is damaged is highly debated, in particular, since the discovery that protooncogenes and tumor suppressor genes besides regulating signal transduction pathways involved in cell cycle control also affect cellular metabolism $[3,4]$. Nowadays, the idea that metabolic reprogramming is a hallmark of cancer is well established, regardless of whether the cause is or is not a defective respiration $[3,5]$.

In this work, we have analyzed the OXPHOS status relative to glycolysis in a model of mouse skin carcinogenesis formed by four cell lines with increasing tumorigenic abilities. Mouse skin chemical carcinogenesis is a well-characterized model system to study the genetic and biochemical changes associated with tumor 
initiation, promotion and progression. In this model, tumors are induced on the skin of mice by treatment with chemicals, either by the "two-stage" or "complete" carcinogenesis protocols [6]. The two-stage protocol involves treatment with a single dose of a carcinogen; i.e., 7, 12-dimethylbenz(a)anthracene (DMBA) followed by repeated applications of the tumor promoter 12-Otetradecanoylphorbol-13-acetate (TPA), which gives rise to the appearance of benign papillomas. A small fraction of papillomas spontaneously progress to malignant squamous cell carcinomas (SCCs), which lately can undergo an epithelial-mesenchymal conversion and form highly aggressive spindle cell carcinomas (SpCC) [7]. Complete carcinogenesis, on the other hand, involves repeated applications of the carcinogen DMBA. Here, DMBA-induced cell lines corresponding to different stages of skin carcinogenesis were used to evaluate whether OXPHOS dysfunctions that lead to increased glycolytic activity occur associated with tumor progression.

\section{Materials and Methods}

\subsection{Cell lines, culture conditions and Gal/Glu index determination}

MCA3D cells were derived by treatment of a primary mouse epidermal keratinocyte culture with DMBA, followed by selection in high calcium medium that induces terminal differentiation of normal keratinocytes [8]. PB cells were obtained from a papilloma induced by two-stage carcinogenesis [9]. The A5 cell line was derived from the spindle component of a skin carcinoma induced by complete carcinogenesis [10], and CarC cells were explanted from a carcinoma 
induced by two-stage carcinogenesis [11]. A summary of the origin and characteristics of the cell lines is presented in Supplementary Material (Table S1). MCA3D and PB cells have normal H-Ras genes, while both CarC and A5 cell lines exhibit an identical mutation at codon 61 of $H$-Ras [8,11,12]. Moreover, CarC cells show complete loss of the normal H-Ras allele [11] and have the Ink4 locus encoding the cell cycle regulators $\mathrm{p} 15^{\text {Ink4b }}, \mathrm{p} 16^{\text {Ink4b }}$ and $\mathrm{p} 19^{\text {Arf }}$ deleted [13]. A5 cells, on the other hand, have inactivated the tumor suppressor Trp53 gene encoding p53 [10], whereas CarC has normal Trp53 [14]. Cell lines were cultured in Dulbecco's modified Eagle's medium (DMEM)- Ham's F12 medium (1:1) supplemented with $10 \%$ foetal bovine serum, $2 \mathrm{mM}$ L-glutamine, and antibiotics: $100 \mu \mathrm{g} / \mathrm{ml}$ ampicillin, $60 \mu \mathrm{g} / \mathrm{ml}$ gentamicin and $2.5 \mu \mathrm{g} / \mathrm{ml}$ amphotericin B. Cells were grown at $37^{\circ} \mathrm{C}$ in a $5 \% \mathrm{CO}_{2}$-humidified atmosphere.

To compare the cell abilities to grow in the presence of either glucose or galactose as a sugar source, cells were transferred to sugar-free DMEM medium (Gibco) containing glucose $(4.5 \mathrm{~g} / \mathrm{l})$ or galactose $(0.9 \mathrm{~g} / \mathrm{l})$ plus the supplements cited above. Cells were incubated up to 8 days, refreshing the medium every 2 days. Doubling times were calculated by applying the suitable formula DT $=\log 2 \mathrm{x}$ $\left(t-t_{0}\right) / \log N-\log N_{0}$, where $\left(t-t_{0}\right)$ is the total days of incubation and $N_{0}$ and $N$, the number of cells at the beginning and end of incubation, respectively. The Gal/Glu index is the ratio of the corresponding doubling times.

\subsection{Tumorigenicity assay}


Cells were collected, washed with PBS (without calcium) and intradermally/subcutaneously injected at the two flanks of 8 week-old Balb/c female athymic nude mice (Harlan). Each animal received approximately $2 \times 10^{6}$ cells/site. Tumor development was measured about twice per week and the size of tumors calculated from calliper measurements of two orthogonal diameters.

\subsection{Western-blot analysis and BEC index determination}

Collected cells were centrifuged and pellets frozen at $-20^{\circ} \mathrm{C}$ until use. Pellets were lysed in cold RIPA buffer supplemented with complete protease inhibitors cocktail (Roche). The homogenates were kept in ice for at least $15 \mathrm{~min}$ and, if necessary, passed through a needle $(0.4 \times 13 \mathrm{~mm})$ to clarify the extracts before centrifuging at $13,000 \mathrm{rpm}$ and $4^{\circ} \mathrm{C}$ for $10 \mathrm{~min}$. The supernatants were saved and the protein content determined with the BCA protein assay kit (Pierce). Extracts were stored frozen until use.

Protein extracts $(20 \mu \mathrm{g})$ were separated in 10\% SDS-PAGE gels and electrotransferred to either Immobilon-P or nitrocellulose membranes (Millipore). In order to determine the BEC index, membranes were probed with a polyclonal antibody against $\beta$-ATPase [15] and, subsequently, with monoclonal antibodies recognizing Hsp60 and GAPDH (Stressgen). Appropriate secondary antibodies coupled to horseradish peroxidase were used, and peroxidase activity was developed using either an enhanced chemoluminiscence (ECL) kit (Amersham) or the Odissey detection system (nitrocellulose filters). The signals obtained for each enzyme were quantified by densitometric analysis with Image J program. The BEC 
index was calculated dividing the ratio $\beta$-ATPase/Hsp60 by the value obtained for GAPDH [2].

Determination of protein levels of the respiratory chain subunits was achieved as indicated above using $50 \mu \mathrm{g}$ protein extracts and monoclonal antibodies specific for COXI and NDUFA9 (Mitosciences). A monoclonal antibody against $\beta$-actin (Sigma) was used as loading control.

2.4. Determination of the activity and Blue Native electrophoresis of the respiratory chain complexes

Enzymatic activity measurements were performed with homogenates obtained from $2 \times 10^{6}$ cells grown as described in section 2.1, washed in PBS and resuspended and sonicated in SETH buffer (250 mM sucrose, 2 mM EDTA, $100 \mathrm{U} / \mathrm{L}$ Heparine, $10 \mathrm{mM}$ Tris- $\mathrm{HCl}, \mathrm{pH}$ 7.4). The amount of protein was determined using the DC Protein Assay kit (Bio-Rad). The activities of the respiratory chain complexes I, II, III and IV and the mitochondrial mass marker citrate synthase were measured by spectrophotometric methods as previously described [16].

Blue native electrophoresis was performed as previously reported [17]. Mitochondrial protein extracts were obtained by treatment of the cells with $2 \mathrm{mg} / \mathrm{ml}$ digitonin followed by solubilization of the native complexes with $2 \%$ lauryl maltoside. Mitochondrial extracts (40 $\mu \mathrm{g}$ ) were loaded on native $4-15 \%$ polyacrilamide gradient gels. After electrophoresis, proteins were transferred to a PVDF membrane (Millipore) and probed with the specific complex I monoclonal antibody NDUFA9 (Mitosciences). 
In-gel activity assays were performed as described [17]. For complex I activity assay, gels were incubated $1-2 \mathrm{~h}$ at $37^{\circ} \mathrm{C}$ in $2 \mathrm{mM}$ Tris- $\mathrm{HCl}, \mathrm{pH} 7.4,0.1 \mathrm{mg} / \mathrm{ml}$ $\mathrm{NADH}$, and $2.5 \mathrm{mg} / \mathrm{ml}$ nitrotetrazolium blue (NTB). Stained gels were washed with distilled water and scanned.

\subsection{ATP steady state levels}

ATP measurements were carried out as previously described [18], with some modifications. Briefly, $2 \times 10^{5}$ cells were grown for 24 hours in glu/gal medium in $35 \mathrm{~mm}$ dishes to approximately $80 \%$ confluence and then incubated for 2.5 hours in a solution $\left(156 \mathrm{mM} \mathrm{NaCl}, 3 \mathrm{mM} \mathrm{KCl}, 2 \mathrm{mM} \mathrm{MgSO}_{4}, 1,25 \mathrm{mM} \mathrm{KH}_{2} \mathrm{PO}_{4}, 2\right.$ $\mathrm{mM} \mathrm{CaCl} 2,20 \mathrm{mM}$ HEPES, $\mathrm{pH}$ 7,35) containing a combination of substrates and inhibitors: $5 \mathrm{mM}$ 2-deoxy-D-glucose/ $1 \mathrm{mM}$ pyruvate (for oxidative ATP) and 2deoxy-D-glucose/pyruvate/oligomycin (for residual ATP). After incubation, cells were washed with PBS and lysed with $1 \mathrm{~mL}$ of boiling $100 \mathrm{mM}$ Tris, 4 mM EDTA, $\mathrm{pH}$ 7.75. Lysates were collected and incubated for two minutes at $100^{\circ} \mathrm{C}$, centrifuged $10 \mathrm{~min}$ at $13.000 \mathrm{x} \mathrm{g}$ and $4^{\circ} \mathrm{C}$ and supernatants frozen. ATP measurement was carried out with an Optocomp I luminometer (MGM instruments Inc), using the ATP bioluminescence assay kit CLS II (Roche Applied Science) according to manufacturer's instructions. ATP levels were calculated with a standard ATP/luminescence curve and normalized by the total amount of protein measured with the Micro BCA Protein Assay Kit (Thermo Scientific). Assays were performed by duplicate in four independent experiments.

\subsection{Measurement of ROS generation}


The production of cytoplasmic and mitochondrial ROS was assessed using dichlorodihydrofluorescein diacetate (H2DCFDA, Molecular Probes) and MitoSOX $^{\mathrm{TM}}$ red (Invitrogen), respectively.

For $\mathrm{DCH}_{2} \mathrm{~F}-\mathrm{DA}$ assays, cells $\left(1 \times 10^{6}\right)$ were grown for $48 \mathrm{~h}$ in "glucose/galactose medium" (DMEM plus 5\% foetal bovine serum, 2 g/l glucose, $2.5 \mathrm{~g} / \mathrm{l}$ galactose, $100 \mathrm{U} / \mathrm{ml}$ penicillin and $100 \mu \mathrm{g} / \mathrm{ml}$ streptomycin). Cells were collected, washed in PBS and counted. Approximately $5 \times 10^{5}$ cells were incubated in the absence or presence of $10 \mu \mathrm{M}$ 2'7-dichlorodihidrofluorescein diacetate $\left(\mathrm{DCH}_{2} \mathrm{~F}-\mathrm{DA}, \mathrm{Molecular}\right.$ Probes) in PBS, at $37^{\circ} \mathrm{C}$ for $30 \mathrm{~min}$ in the dark. After incubation, cells were washed, resuspended in $0.5 \mathrm{ml}$ PBS, and immediately analysed with a FACScan flow cytometer (Beckton Dickinson). Mean fluorescence intensity was determined with CellQuest software (Beckton Dickinson).

For measurement of mitochondrial ROS, $0.75 \times 10^{5}$ cells were grown in glu/gal medium. After addition of $5 \mu \mathrm{M}$ MitoSOX ${ }^{\mathrm{TM}}$ red and incubation at $37^{\circ} \mathrm{C}$ for $30 \mathrm{~min}$ in the dark, cells were collected in $0.5 \mathrm{ml}$ glu/gal medium and immediately analysed with a FC500 MPL flow cytometer (Beckman Coulter). Forward and side scatter were used to gate the viable population of cells, and mean fluorescence intensity was determinate with MXP software (Beckman Coulter). Experiments were performed by triplicate in three independent experiments.

\section{Results and Discussion}




\subsection{Tumorigenic behavior of the cell lines}

We tested the tumorigenic capacities of the cell lines by transplantation in nude mice. The kinetics of tumor growth and the tumorigenic properties of the cell lines are shown in Fig 1. As can be seen, MCA3D cells were nontumorigenic. In contrast, PB cells induced tumors in about $67 \%$ of injection sites that required relatively long latency periods. A5 and CarC cell lines, on the other hand, were highly tumorigenic inducing tumors at all injection sites with short latency periods and similar kinetics of tumor growth, although CarC tumors appeared earlier (Fig. 1A, B). Tumors induced by PB were histologically typed as moderately to welldifferentiated SCCs [9], whereas those induced by A5 and CarC were highly undifferentiated spindle cell carcinomas $[10,11,14]$.

\subsection{Mitochondrial function and tumorigenicity: BEC index and OXPHOS activity}

Cuezva and collaborators defined the BEC index $(\beta-$ F1-ATPase/GADPH protein ratio) as a bioenergetic mitochondrial index relative to the cellular glycolytic potential [2]. In patients with lung adenocarcinomas, a reduced BEC index correlated with worse prognosis [19]. In order to ascertain whether similar alterations of this bioenergetics signature occurred in our experimental model, we determined BEC index values for MCA3D, PB, A5 and CarC cell lines. As shown in Figure 2A, a clear decrease in the BEC index, ranging from 1.93 in nontumorigenic MCA3D to 0.53 in highly malignant CarC cells, was detected associated with enhanced tumorigenicity. To determine whether this reduction was due to an impaired OXPHOS function, the activity of the respiratory chain complexes was 
measured in the different cell lines by spectrophotometric assays, as indicated in Materials and methods. No significant differences were found in the activities of the OXPHOS complexes (data not shown). The activity of the complexes was also measured using in-gel activity assays. The activity of complex I together with the levels of fully assembled complex observed in Blue Native gels are shown in Fig. 2B. Also, the expression of proteins markers for complexes I and IV, as determined by Western blotting, is shown in Fig. 2C. No significant differences in these parameters were found between the different cell lines, in accordance with the spectrophotometric analysis.

The gradual decline in the BEC signature associated with enhanced tumorigenicity observed in our cell lines is in line with the proposal of reduced BEC index as a bad prognostic factor in assessing clinical outcome for cancer patients $[2,19]$. Apparently, this decrease is not caused by significant changes in the protein composition or activity of the mitochondrial respiratory chain complexes.

\subsection{Glycolisis and tumorigenicity: Gal/Glu index}

Since the activity of the respiratory chain complexes appeared not to be affected in tumorigenic cell lines, the observed reduction in the BEC index could be explained by an increased glycolytic activity or by a defective mitochondrial function not observed in the measurements of the activity of the OXPHOS complexes. To test these possibilities, we measured the relative ability of the different cell lines to grow in galactose versus high glucose medium (Gal/Glu index). Galactose is not utilized efficiently as a glycolytic substrate; therefore only 
cells with a fully functional OXPHOS system grow in these conditions [20]. Cells with efficient OXPHOS grow equally well in media supplemented with either galactose or glucose.

The doubling times of MCA3D, $\mathrm{PB}, \mathrm{A} 5$ and CarC cells growing in the presence of either galactose or glucose were measured and the Gal/Glu indexes calculated. We observed an increase in the Gal/Glu index, ranging from 1.12 in MCA3D to 1.67 in CarC, which correlated with the tumorigenic potential of the cell lines (Fig. 3). Interestingly, cells grew poorly in the presence of galactose, and the observed increase in Gal/Glu index was mainly due to the most efficient growth of tumorigenic cell lines in high glucose medium.

To further investigate the OXPHOS capacity of the cells, we evaluated the ATP contents when the cells grew in 2-deoxyglucose, condition in which ATP is exclusively produced through OXPHOS (Figure 3B). Interestingly the ATP levels were similar in all cases, thus indicating that the four cell lines can produce equivalent amounts of ATP via OXPHOS. The mitochondrial origin of ATP is clearly demonstrated with the incubation in the presence of oligomycin, an inhibitor of ATP synthase, which abolished the synthesis completely.

Taken together, the variations in BEC and Gal/Glu indexes observed in our cell model likely reflect a progressive metabolic adaptation from OXPHOS to glucose fermentation associated with increased malignancy in neoplastic cells. This metabolic change appears not to be caused by impairment of mitochondrial respiration as the capacity to synthesize ATP via OXPHOS and to grow in galactose medium are comparable in all cell lines studied. 


\subsection{ROS generation and tumorigenicity}

Mitochondria are considered the main source of reactive oxygen species (ROS), and ROS production is generally associated with impairments of the respiratory chain [21]. Therefore, in order to ascertain whether alterations in the generation of ROS also occur associated with tumorigenicity, we measured the intracellular ROS levels in the different cell lines. A shift in the generation of ROS was found when comparing nontumorigenic MCA3D cells with tumorigenic PB, A5 and CarC cell lines (Fig. 4). Tumorigenic cell lines produced similar amounts of ROS that were higher (about threefold) than the amount of ROS generated by MCA3D. Since papilloma-derived PB is only moderately tumorigenic and spindle carcinoma cell lines A5 and CarC represent advanced stages of skin carcinogenesis [7], this finding suggests that oxidative stress increases early during tumor progression.

To learn of the intracellular source of ROS, we determined the levels of mitochondrial superoxide anion using MitoSOX, a specific mitochondrial ROS reagent. Interestingly, the results found (Fig S1) were similar in the different cell lines except in the case of PB that showed a very low level of superoxide, indicating that ROS production in this particular cell line mostly occurs at a site different from the mitochondria, likely the endoplasmic reticulum and/or the plasma membrane. Likewise, since the levels of mitochondrial superoxide anion of A5 and CarC are similar to that of MCA3D, the increase observed in the total ROS levels produced by the former cell lines with respect to the latter (Fig. 4) seems to be generated outside the mitochondria. 
In summary, the relationship between oxidative function, glycolysis and tumorigenicity was analyzed in four epidermal cell lines representing an in vitro model of tumor progression, in which two indexes, BEC and Gal/Glu, were determined. In both cases, a gradual increase in glycolytic activity compared to OXPHOS function was observed associated with tumorigenicity. Notwithstanding, alterations in the expression or activity of components of the respiratory chain complexes were not found. In agreement, the corresponding mitochondrial ATP synthetic abilities of the cell lines were similar. These results agree well with recent ideas suggesting that mitochondria are not necessarily damaged in cancer cells, but cellular metabolism is reprogrammed from mitochondrial respiration to aerobic glycolysis in order to support anabolic growth. Thus, the ATP required for growth is mainly obtained through inefficient glycolysis that otherwise generates increased levels of biosynthetic precursors necessary to sustain a high rate of cell proliferation $[3,4,5]$. The mechanisms involved in the metabolic adaptation of cancer cells are still poorly understood but they seem to be directed by alterations in proto-oncogenes and tumor suppressor genes. The cell model presented in this work represents a suitable tool to investigate the molecular mechanisms leading to the metabolic reprogramming of cancer cells.

\section{Acknowledgments}

This work was supported by grants of the Center for Biomedical Research on Rare Diseases (CIBERER), Instituto de Salud Carlos III (grants PI 07/0167 and PI 10/0703 to RG), Comunidad Autónoma de Madrid (grant number GEN-0269 to 
RG and grant S2010/BMD-2359, SkinModel-CM, to MQ) and the Spanish Ministry of Science and Innovation (grant SAF2010-19152 to MQ).

\section{References}

[1] O. Warburg, F. Wind, E. Negelein, The Metabolism of Tumors in the Body, J Gen Physiol 8 (1927) 519-530.

[2] J.M. Cuezva, M. Krajewska, M.L. de Heredia, S. Krajewski, G. Santamaria, H. Kim, J.M. Zapata, H. Marusawa, M. Chamorro, J.C. Reed, The bioenergetic signature of cancer: a marker of tumor progression, Cancer Res 62 (2002) $6674-6681$.

[3] W.H. Koppenol, P.L. Bounds, C.V. Dang, Otto Warburg's contributions to current concepts of cancer metabolism, Nat Rev Cancer 11 (2011) 325-337.

[4] P.S. Ward, C.B. Thompson, Metabolic reprogramming: a cancer hallmark even warburg did not anticipate, Cancer Cell 21 (2012) 297-308.

[5] D. Hanahan, R.A. Weinberg, Hallmarks of cancer: the next generation, Cell 144 (2011) 646-674.

[6] E.L. Abel, J.M. Angel, K. Kiguchi, J. DiGiovanni, Multi-stage chemical carcinogenesis in mouse skin: fundamentals and applications, Nat. Protoc. 4 (2009) 1350-1362.

[7] R.J. Akhurst, A. Balmain, Genetic events and the role of TGF beta in epithelial tumour progression, J Pathol 187 (1999) 82-90.

[8] M. Kulesz-Martin, A.E. Kilkenny, K.A. Holbrook, V. Digernes, S.H. Yuspa, Properties of carcinogen altered mouse epidermal cells resistant to calciuminduced terminal differentiation, Carcinogenesis 4 (1983) 1367-1377. 
[9] S.H. Yuspa, D. Morgan, U. Lichti, E.F. Spangler, D. Michael, A. Kilkenny, H. Hennings, Cultivation and characterization of cells derived from mouse skin papillomas induced by an initiation-promotion protocol, Carcinogenesis 7 (1986) 949-958.

[10] P.A. Burns, C.J. Kemp, J.V. Gannon, D.P. Lane, R. Bremner, A. Balmain, Loss of heterozygosity and mutational alterations of the p53 gene in skin tumours of interspecific hybrid mice, Oncogene 6 (1991) 2363-2369.

[11] A. Buchmann, B. Ruggeri, A.J. Klein-Szanto, A. Balmain, Progression of squamous carcinoma cells to spindle carcinomas of mouse skin is associated with an imbalance of H-ras alleles on chromosome 7, Cancer Res 51 (1991) 4097-4101.

[12] M. Quintanilla, S. Haddow, D. Jonas, D. Jaffe, G.T. Bowden, A. Balmain, Comparison of ras activation during epidermal carcinogenesis in vitro and in vivo, Carcinogenesis 12 (1991) 1875-1881.

[13] S. Linardopoulos, A.J. Street, D.E. Quelle, D. Parry, G. Peters, C.J. Sherr, A. Balmain, Deletion and altered regulation of p16INK4a and p15INK4b in undifferentiated mouse skin tumors, Cancer Res 55 (1995) 5168-5172.

[14] M. Pons, J.C. Cigudosa, S. Rodriguez-Perales, J.L. Bella, C. Gonzalez, C. Gamallo, M. Quintanilla, Chromosomal instability and phenotypic plasticity during the squamous-spindle carcinoma transition: association of a specific $\mathrm{T}(14 ; 15)$ with malignant progression, Oncogene 24 (2005) 7608-7618.

[15] P. Pena, R. Garesse, The beta subunit of the Drosophila melanogaster ATP synthase: cDNA cloning, amino acid analysis and identification of the protein in adult flies, Biochem Biophys Res Commun 195 (1993) 785-791. 
[16] M. Perez-Carreras, P. Del Hoyo, M.A. Martin, J.C. Rubio, A. Martin, G. Castellano, F. Colina, J. Arenas, J.A. Solis-Herruzo, Defective hepatic mitochondrial respiratory chain in patients with nonalcoholic steatohepatitis, Hepatology 38 (2003) 999-1007.

[17] M.A. Calvaruso, J. Smeitink, L. Nijtmans, Electrophoresis techniques to investigate defects in oxidative phosphorylation, Methods 46 (2008) 281287.

[18] M. McKenzie, D. Liolitsa, N. Akinshina, M. Campanella, S. Sisodiya, I. Hargreaves, N. Nirmalananthan, M.G. Sweeney, P.M. Abou-Sleiman, N.W. Wood, M.G. Hanna, M.R. Duchen, Mitochondrial ND5 gene variation associated with encephalomyopathy and mitochondrial ATP consumption, J Biol Chem 282 (2007) 36845-36852.

[19] J.M. Cuezva, G. Chen, A.M. Alonso, A. Isidoro, D.E. Misek, S.M. Hanash, D.G. Beer, The bioenergetic signature of lung adenocarcinomas is a molecular marker of cancer diagnosis and prognosis, Carcinogenesis 25 (2004) 1157-1163.

[20] B.H. Robinson, Use of fibroblast and lymphoblast cultures for detection of respiratory chain defects, Methods Enzymol 264 (1996) 454-464.

[21] M. Rigoulet, E.D. Yoboue, A. Devin, Mitochondrial ROS generation and its regulation: mechanisms involved in $\mathrm{H}(2) \mathrm{O}(2)$ signaling, Antioxid Redox Signal 14 (2011) 459-468.

\section{Legends to Figures}


Fig. 1. Tumorigenic characteristics of the cell lines. Cells were injected into the two flanks of nude mice and the size of tumors was measured every 2-3 days as indicated in Materials and methods. (A) Kinetics of tumor growth. The average values $( \pm S D)$ of tumor size are indicated $(P B, n=4 ; A 5, n=10 ; C a r C, n=6)$. (B) Incidence of tumors induced by the cell lines. The table records the number of tumors developed (higher than $0.5 \mathrm{~cm}^{2}$ ) per total number of injection sites. MCA3D cells gave rise to small cysts below $0.5 \mathrm{~cm}^{2}$ that regressed or remained stable at the end of the experiment (40 days postinjection). The latency period was estimated as the time needed for tumors to reach a size of $0.5 \mathrm{~cm}^{2}$. SCC, squamous cell carcinoma; SpCC, spindle cell carcinoma.

Fig 2. BEC index decreases with tumorigenicity. (A) The expression of $\beta$-ATPase, Hsp60 and GAPDH was determined by Western blotting. BEC indexes in the table below were calculated as shown in Materials and methods and are expressed as the average $( \pm S D)$ values obtained from five independent experiments. Statistical analysis was performed by the Student's $t$-test. Significant differences with respect to MCA3D were observed. ${ }^{* * *} \mathrm{P}<0.001$.

(B) In-gel activity assay and blue-native electrophoresis of complex I was performed as described in Materials and Methods. (C) Analysis of Cox I (complex V) and NDUFA9 (complex I) expression by Western blotting. $\beta$-actin was used as a loading control.

Fig 3. (A) Gal/Glu index increases with tumorigenicity. Graphs represent the number of cells versus the incubation period in medium containing either glucose or galactose; a representative experiment is shown. Gal/Glu indexes in the table at 
the bottom were calculated as the average $( \pm$ SD) of duplicate determinations of 35 independent experiments. Statistical analysis was performed by the Student's $t$ test. Significant differences with respect to MCA3D were observed in A5 and CarC. ${ }^{*} \mathrm{P}<0.02$. (B) Cell lines synthesize similar oligomycin-sensitive ATP steady state levels. The ATP synthesized by the cell lines was determined in the presence of 2deoxyglucose as described in Materials and methods. The mitochondrial origin of the measured ATP was authenticated by the complete inhibition of its production with oligomycin.

Fig 4. Increased ROS generation is an early event in tumorigenesis. The mean fluorescence intensity of a representative experiment is shown in the absence (gray) and presence (blue) of reagent. The diagram at the bottom represents the mean values $( \pm$ SD) of ROS levels plotted from triplicate determinations of four independent experiments. Statistical analysis was performed by the Student's $t$ test. ${ }^{* *} \mathrm{P}<0.01,{ }^{*} \mathrm{P}<0.05$.

Fig S1. Mitochondrial ROS production in the different cell lines. Cells were incubated in the presence of MitoSOX and the resulting fluorescence intensity measured as described in Materials and methods. The diagram represents the mean values $( \pm S D)$ of ROS levels plotted from triplicate determinations of three independent experiments. Statistical analysis was performed by the Student's $t$ test. Significant differences were observed in PB versus MCA3D. No significant differences were observed in the levels of MCA3D with respect to the other two cell lines. ${ }^{* * *} \mathrm{P}<0.001$. 


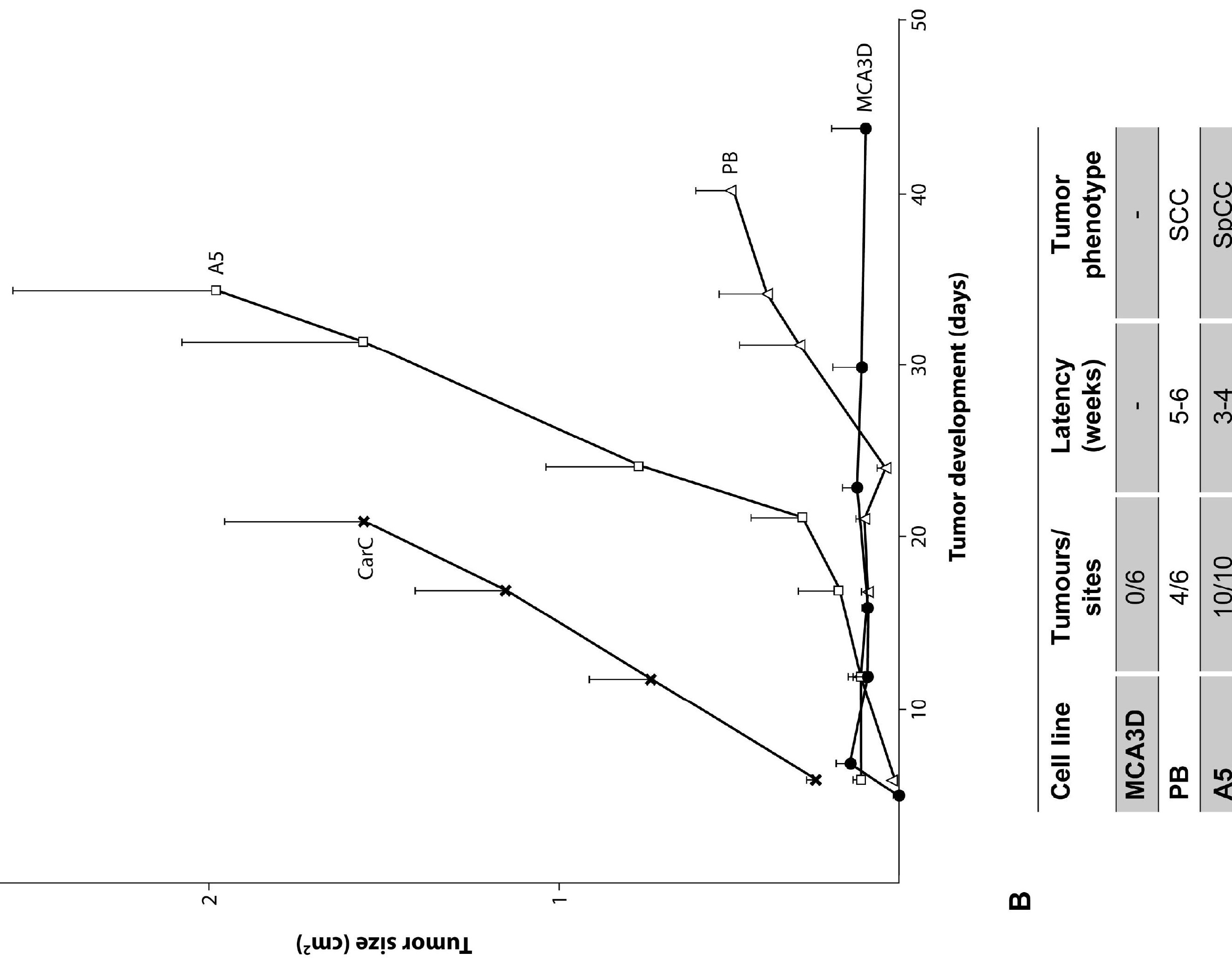




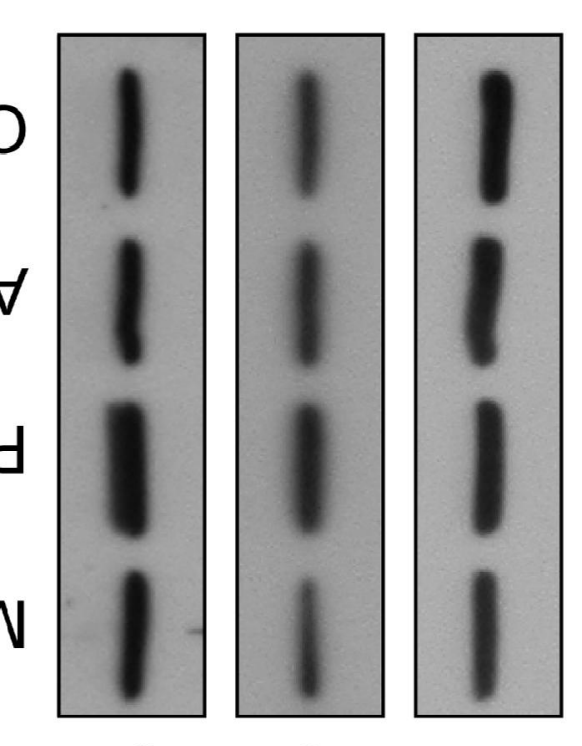

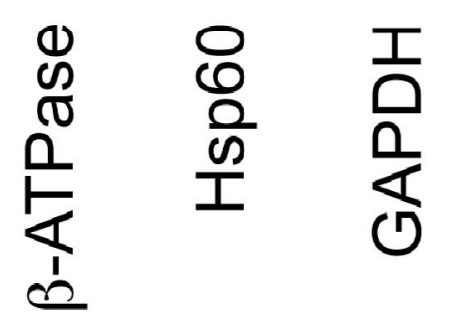

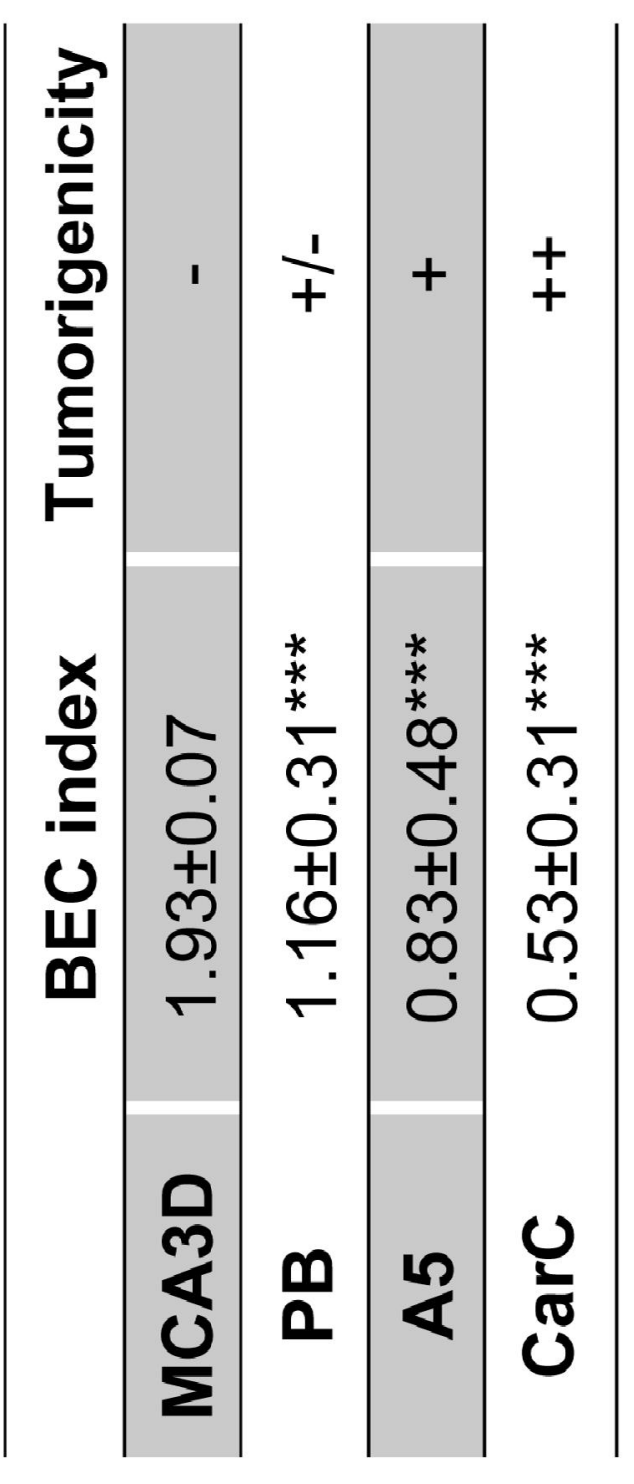

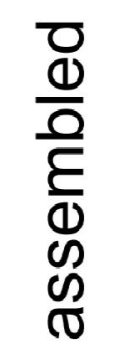

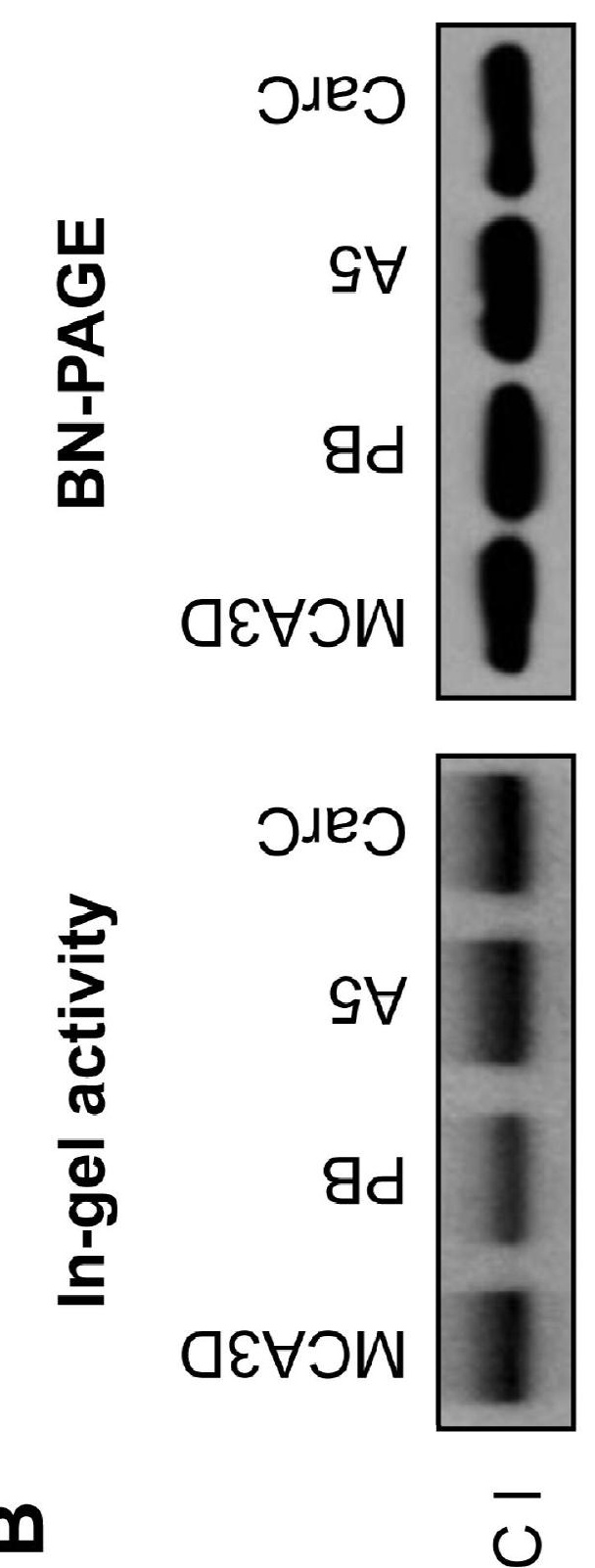



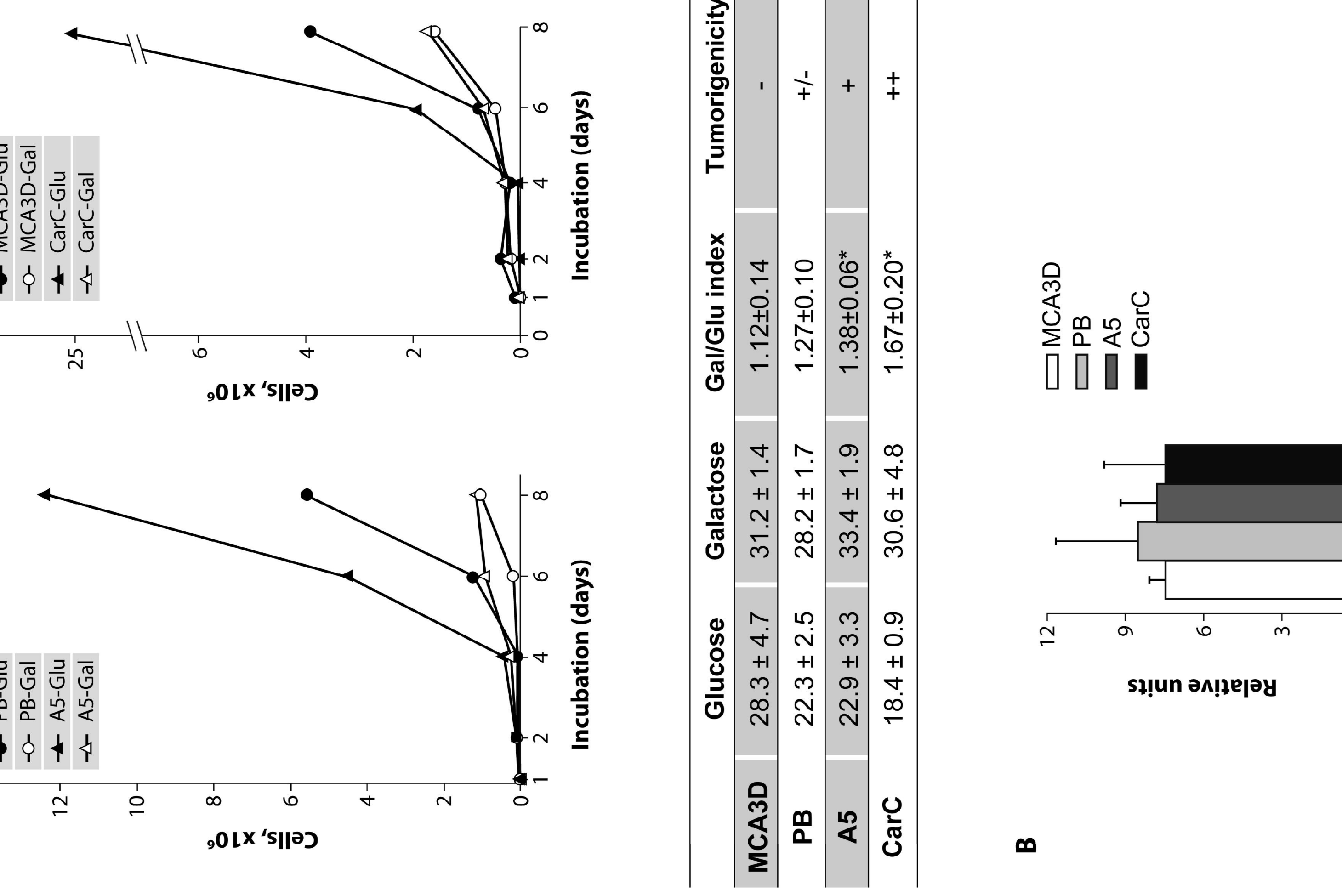

sł!un әм!де|әу

$\infty$ 
Table S1. Summary of cell lines characteristics

\begin{tabular}{llcccc}
\hline Cell line & \multicolumn{1}{c}{ Origin } & Phenotype & $\begin{array}{l}\text { H-Ras } \\
\text { mutation }\end{array}$ & $\begin{array}{l}\text { Trp53 } \\
\text { inactivation }\end{array}$ & $\begin{array}{l}\text { Ink4 } \\
\text { deletion }\end{array}$ \\
\hline MCA3D & DMBA-treated primary keratinocytes & E & No & No & No \\
PB & DMBA/TPA-induced papilloma & E & No & ND & Yes \\
A5 & DMBA-induced carcinoma & F & Codon 61 & No & Yes \\
CarC & DMBA/TPA-induced carcinoma & F & Codon 61 & Loss of normal allele &
\end{tabular}

DMBA, 7, 12-dimethylbenz(a)anthracene; TPA, 12-O-tetradecanoylphorbol-13-acetate; E, epithelial; F, fibroblastic; ND, not determined 


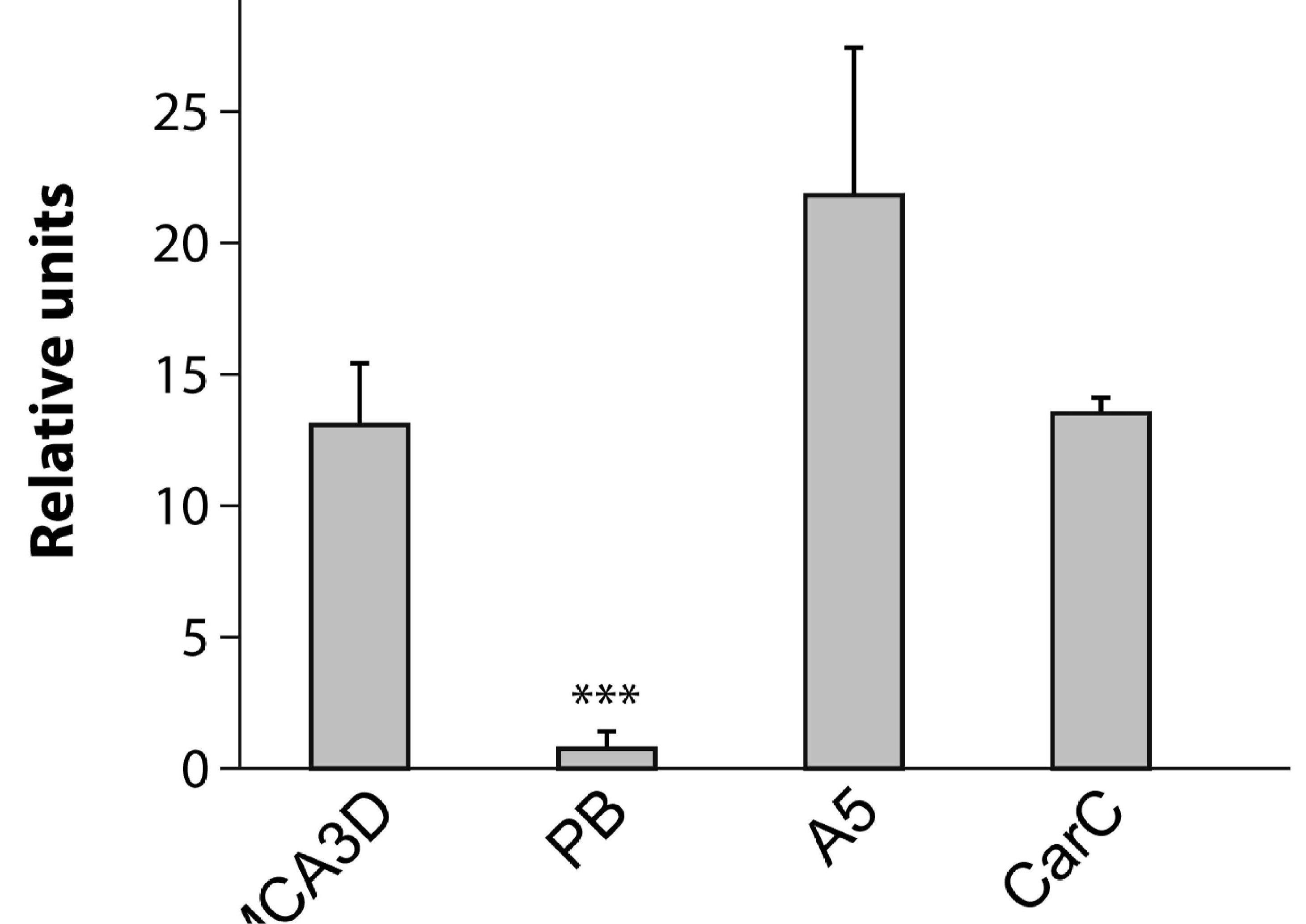

